\title{
A two-scale contact model for collisions between blocks in CDEM
}

\author{
LI ShiHai, TANG DeHong* \& WANG Jie \\ Institute of Mechanics, Chinese Academy of Sciences, Beijing 100190, China
}

Received April 22, 2015; accepted July 20, 2015; published online August 3, 2015

\begin{abstract}
Contact detection between interacting blocks is of great importance to discontinuity-based numerical methods, such as DDA, DEM, and NMM. A rigorous contact theory is a prerequisite to describing the interactions of multiple blocks. Currently, the penalty method, in which mathematical springs with high stiffness values are employed, is always used to calculate the contact forces. High stiffness values may cause numerical oscillations and limit the time step. Furthermore, their values are difficult to identify. The intention of this study is to present a two-scale contact model for the calculation of forces between colliding blocks. In this new model, a calculation step taken from the moment of contact will be divided into two time stages: the free motion time stage and the contact time stage. Actually, these two time stages correspond to two real physical processes. Based on this, we present a new numerical model that is intended to be more precise and useful in calculating the contact forces without mathematical springs. The propagation of the elastic wave during collision is of a characteristic length, which determines the volume of material involved in the contact force calculation. In conventional contact models, this range is always regarded as the length of one element, which may lead to an inaccurate calculation of contact forces. In fact, the real scale of this range is smaller than the length of a single element, and subdivided elements, which are refined according to the characteristic length and are presented in the new contact model.
\end{abstract}

two-scale, contact model, collision, elastic wave, element subdivision

Citation: $\quad$ Li S H, Tang D H, Wang J. A two-scale contact model for collisions between blocks in CDEM. Sci China Tech Sci, 2015, 58: 1596-1603, doi: $10.1007 / \mathrm{s} 11431-015-5902-4$

\section{Introduction}

The description of the mechanical features of the collision between interacting blocks is typically a complex problem in any discontinuity-based numerical method, such as the discrete element method (DEM) [1-3], discontinuous deformation analysis (DDA) [4-7], the combined finite-discrete element method (FDEM) [8,9], and the numerical manifold method (NMM) [10,11]. Using an appropriate contact model is the key to resolving this problem, which is the calculation of contact forces during dynamic collision. For conventional contact theory, the contact forces are always calculated using springs with high stiffness

\footnotetext{
*Corresponding author (email: tsinghtdh@126.com)
}

values. Furthermore, the calculation of the Hertz force $[12,13]$ between two elastic balls is a relatively advanced method.

The most general method used in the calculation of contact forces is the application of springs between two contact points, and the types of springs are based on the contact types. The penalty method is regarded as an efficient approach to solving this problem and has been used in many DDA and DEM models [14-19]. In the penalty method, contacting blocks are connected by springs with high stiffness values, and the displacement compatibility condition may be satisfied through the continuum condition. Goodman et al. [19] presented a useful element to calculate the stiffness values of the contact springs. It is important to note that the mechanical properties of the block system are al- 
tered if springs are added into the calculation. In this manner, the structure layer model [20], which is cut from the adjacent element, may be used to solve the aforementioned problem. However, the thickness of the layer is not explicitly defined.

The model presented in this study is intended to be a useful tool for the calculation of contact forces during collision. For this model, one calculation step taken from the contact moment will be divided into two time stages: A free motion time stage and a contact time stage. Moreover, the assumption of a perfect inelastic collision is employed at the beginning of the colliding process. Unified node algorithms are used to calculate the motion of the contact points, and the criteria for node separation are also presented. Based on this, the concept of subdivided elements is presented to describe the process of elastic wave propagation within the colliding element.

\section{Basic theory of CDEM}

The continuous-discontinuous element method (CDEM) is an explicit time-history analysis approach for finite difference principles, and a forward difference approximation is adopted to calculate the progressive process through a time-marching scheme. CDEM is the combination of the finite element method (FEM) and discrete element method (DEM), which contain two types of elements, blocks and contacts [21].

The equations of motion are obtained from equilibrium conditions of all forces acting on the nodal masses, resulting in a system of equations of the form,

$$
\boldsymbol{M} \ddot{\boldsymbol{u}}+\boldsymbol{C} \dot{\boldsymbol{u}}+\boldsymbol{F}(t)=\boldsymbol{P}(t),
$$

where $\boldsymbol{u}, \dot{\boldsymbol{u}}$, and $\ddot{\boldsymbol{u}}$ denote vectors containing the nodal displacements, velocities, and accelerations, respectively. $\boldsymbol{M}$ and $\boldsymbol{C}$ are the mass and damping matrices, and the vectors $\boldsymbol{F}(t)$ and $\boldsymbol{P}(t)$ contain the internal and external nodal forces.

To solve the large rotation and deformation of the blocks, the incremental method should be adopted, and strain matrix $[\boldsymbol{B}]$ (instead of stiff matrix $[\boldsymbol{K}]$ ) should be used to calculate the deformation forces. Furthermore, the strain matrix should be renewed at each time step. The main equations used to calculate nodal forces using a strain matrix incremental method are written as

$$
\left\{\begin{array}{l}
{[\Delta \boldsymbol{\varepsilon}]_{i}=[\boldsymbol{B}]_{i}\{\Delta \boldsymbol{u}\}_{e},} \\
{[\Delta \boldsymbol{\sigma}]_{i}=[\boldsymbol{D}][\Delta \boldsymbol{\varepsilon}]_{i},} \\
{[\boldsymbol{\sigma}(\boldsymbol{\tau}+\Delta \boldsymbol{\tau})]_{i}=[\boldsymbol{\sigma}(\boldsymbol{\tau})]_{i}+[\Delta \boldsymbol{\sigma}]_{i},} \\
\{\boldsymbol{F}(\boldsymbol{\tau}+\Delta \boldsymbol{\tau})\}=\sum_{i=1}^{N}[\boldsymbol{B}]_{i}^{\mathrm{T}}[\boldsymbol{\sigma}(\boldsymbol{\tau}+\Delta \boldsymbol{\tau})]_{i} \boldsymbol{w}_{i} \boldsymbol{J}_{i},
\end{array}\right.
$$

where $[\boldsymbol{B}]_{i},[\Delta \boldsymbol{\varepsilon}]_{i},[\Delta \boldsymbol{\sigma}]_{i}, \boldsymbol{w}_{i}$, and $\boldsymbol{J}_{i}$ are the strain matrix, incremental strain, incremental stress, integral coefficient, and
Jacobian determinant, respectively, at the Gaussian point $i$; $[\boldsymbol{D}],\{\Delta \boldsymbol{u}\}_{e}$, and $\{\boldsymbol{F}(\boldsymbol{\tau}+\Delta \boldsymbol{\tau})\}_{e}$ are the elastic matrix, incremental displacement vector, and new node force vector of the element, respectively; and, $N$ is the total number of Gaussian points.

For contact detection between the arbitrary convex polyhedral blocks in the CDEM, a shrunken edge algorithm is employed [21]. In the algorithm, a pair of contacting blocks are identified as a main block and a target block. As shown in Figure 1, shrunken edges are formed by shrunken points on the main block.

Three-dimensional contact detection is then performed by determining the geometric relationship between the shrunken edge and its approaching face on the target block (Figure 2). From the three possible geometric relationships, all six three-dimensional contact types may be precisely identified.

If two-dimensional problems are considered, this algorithm will be simplified. The contact detection process may be performed simply by determining the relationship between vertices on the main block and edges on the target block, as shown in Figure 3. Based on the distance, $d_{n}$, and contact tolerance, $d_{\mathrm{tol}}$, the contact state and contact points may be obtained. After the contact points have been obtained from the detect process, the contact forces, which include normal, shear, and frictional forces, are calculated using incremental displacements and the penalty method.

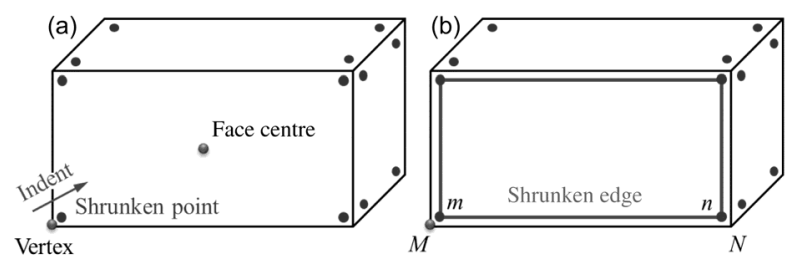

Figure 1 Shrunken edge model. (a) Shrunken points; (b) shrunken edges.

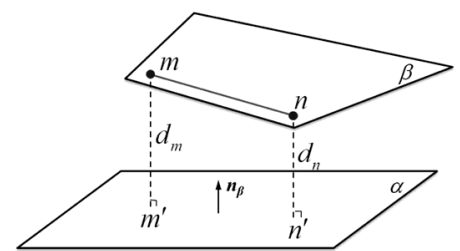

Figure 2 Contact detection between the shrunken edge and target face.

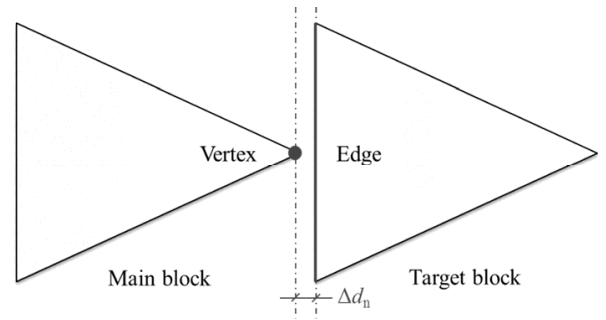

Figure 3 Contact detection between the main vertex and target edge. 


\section{The concept of two time stages}

In the current approach, contact forces are calculated according to the relative incremental displacement between the contact points in a single calculation step. If the two blocks are actually in contact at the beginning of this step, the results are reasonable. However, this condition may not always be satisfied. Considering the numerical discretization of time, it is very common in the calculation that the two blocks are in contact at the end of the step, but are in a separating state at the beginning of the step. In fact, the real physical process for the contact between two blocks consists of two stages: the free motion stage and the contact stage, and only the contact stage will generate contact forces. Figure 4 shows a schematic that describes these two stages.

In the calculation of the embedding value, $\Delta d_{n}$, a hypothesis is employed, in which a target element will not provide counter-forces during the contact stage. The embedding value obtained using this approach is overestimated. In order to decrease the deviation, a smaller contact tolerance and time step should be employed during the calculation. This will have a significant influence on the efficiency of the process.

In order to solve the aforementioned problem, a calculation time step, $\Delta t$, in the contact moment will be divided into two time stages for the new contact model. The free motion time duration is denoted as $\Delta t_{1}$, and the contact time duration as $\Delta t_{2}$. In the meantime, the time step of the other nodes maintains $\Delta t$.

Let $t_{0}$ be the present moment. From time interval $t_{0}$ to $t_{0}+$ $\Delta t_{1}$, it is assumed that $N$ collisions will occur in the entire region, denoted as $I_{1}, I_{2}, \cdots, I_{i}, \cdots, I_{N} . v_{i 0}$ represents the relative velocity of two colliding elements (one is a main element and the other is a target element) at time $t_{0} . c_{m i}$ and $c_{t i}$ represent elastic wave velocities in the main element and target element, respectively. $\Delta t_{i 1}$ and $\Delta t_{i 2}$ represent the time duration of free motion before collision, and the time duration of contact, respectively. The velocity of free movement is assumed constant during $\Delta t_{i 1}$. Take the two-dimensional collision, $I_{i}$, as an example. The procedures for the division of one time step are explained as follows.

(1) At time $t_{0}$, the intending collision, $I_{i}$, which will

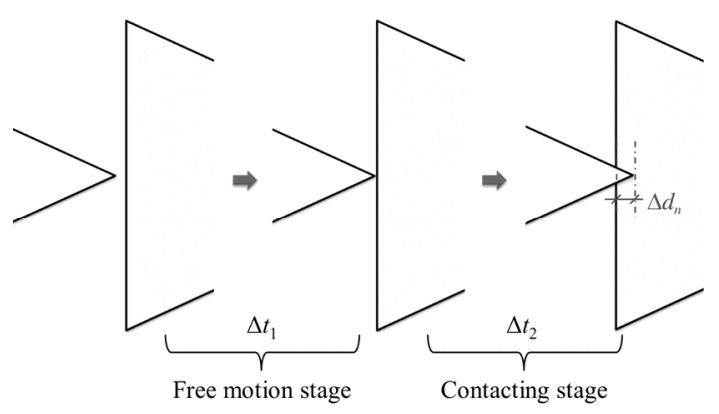

Figure 4 Two physical stages in one calculation step. occur after $\Delta t$ is searched for and predicted based on a contact detection algorithm. As shown in Figure 5, if the distance, $\Delta d_{n}$, is less than the contact tolerance, $d_{\text {tol }}$, the elements will contact.

(2) Based on the geometric position of two colliding elements at times $t_{0}$ and $t_{0}+\Delta t$, the collision curve $L_{i}$ and point $P_{i}$ can be obtained. Here, $P_{i}$ is the intersection point between $L_{i}$ and the edge of the element. Take $P_{i}$ as the initial position of the contact, as shown in Figure 6.

(3) The collision curve, $L_{i}$, will be divided into two segments by $P_{i}$, i.e., $L_{i 1}$ and $L_{i 2}$. The corresponding relationship will be $L_{i 1}=v_{i 0} \Delta t_{i 1}$. As the values of $v_{i 0}$ and $L_{i 1}$ can be obtained, the values of $\Delta t_{i 1}$ and $\Delta t_{i 2}$ can then be calculated. The starting time of the collision will be defined as $t+\Delta t_{i 1}$.

(4) The time step, $\Delta t$, will be used to calculate the movement of nodes that are not in contact with one another. Additionally, $\Delta t_{i 1}$ is used as a time step to calculate the free motion stage of the contact nodes and $\Delta t_{i 2}$ is used as a time step in the contact stage.

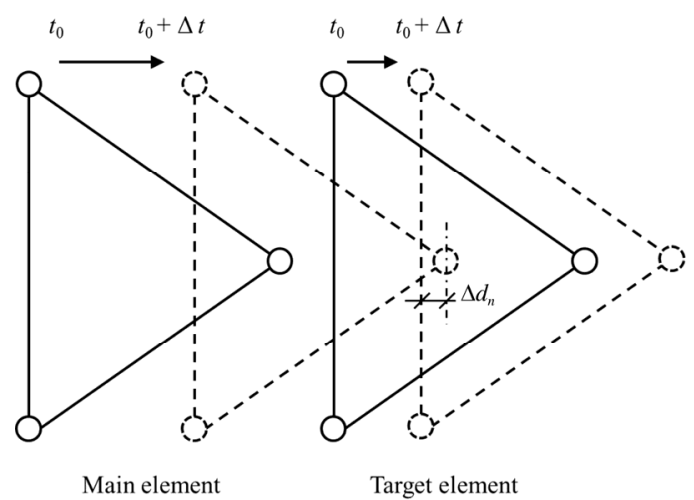

Figure 5 The intending collision between the main block and target block at time $t_{0}+\Delta t$.

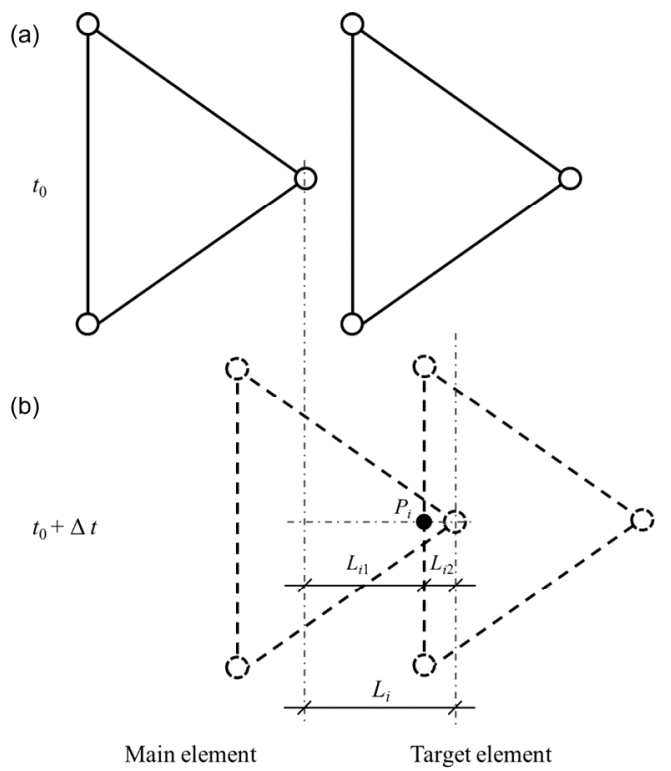

Figure 6 The collision curve $L_{i}$, and point $P_{i}$. (a) Geometric position at times $t_{0}$; (b) geometric position at $t_{0}+\Delta t$. 


\section{The calculation of contact with a unified node}

In many conventional contact models, the penalty method is used to calculate the contact forces. However, the properties of these types of springs are difficult to identify. For springs with high stiffness values requiring a small time step, and because of numerical oscillations, it is not convenient for the efficiency and stability of the contact model. In fact, contact forces are caused by the deformation of target elements than of contact springs. A new solution without contact springs may be developed based on the assumption that the collision of contact points is completely inelastic. This solution contains two stages. The first stage is for interval $t_{0}$ $+\Delta t_{i 1}$ to $t_{0}+\Delta t$, and the second stage is for interval $t_{0}+\Delta t$ to separation time of the contact points.

\subsection{Process for interval $t_{0}+\Delta t_{i 1}$ to $t_{0}+\Delta t$}

To begin with, we assume that no slipping occurs between these two contact points in the interval $t_{0}+\Delta t_{i 1}$ to $t_{0}+\Delta t$. In this manner, the two contact points may be treated as one continuous node with the same velocity. This continuous node may be referred to as a clone node (Figure 7). The mass of the clone node is $M_{i}$ and its corresponding element force vector, $\boldsymbol{F}_{i}$, may be defined as

$$
\left\{\begin{array}{l}
M_{i}=M_{m i}+M_{t i}, \\
\boldsymbol{F}_{i}=\boldsymbol{F}_{m i}+\boldsymbol{F}_{t i},
\end{array}\right.
$$

where $M_{m i}$ and $M_{t i}$ are the masses of the two contact points. $\boldsymbol{F}_{m i}$ and $\boldsymbol{F}_{m i}$ are their respective force vectors.

After collision occurs, the initial condition of the contact points is derived from the momentum conservation equation of completely inelastic collisions, and the fact that the two contact points have the same velocity.

$$
M_{m i} \boldsymbol{v}_{m i 0}+M_{t i} \boldsymbol{v}_{t i 0}=\left(M_{m i}+M_{t i}\right) \boldsymbol{v}_{i},
$$

where $\boldsymbol{v}_{m i 0}$ and $\boldsymbol{v}_{t i 0}$ are the velocity vectors of the two contact points at time $t_{0}$, respectively. $\boldsymbol{v}_{i}$ is the velocity vector of the clone node at $t_{0}+\Delta t_{i 1}$. Since the process of collision is so rapid, the elastic wave is still at the contact point and the other nodes of the element are not affected at $t_{0}+\Delta t_{i 1}$. In addition, conditions remain unchanged during the interval $t_{0}$ to $t_{0}+\Delta t_{i 1}$, so the velocity and stress of the other nodes at $t_{0}$ may be used as initial values. The clone node is treated as a single node until separation occurs.

However, it may be noted that the assumption of completely inelastic collision causes mechanical energy losses, which may be defined as $\Delta E$.

$$
\Delta E=\frac{M_{m i} M_{t i}}{2\left(M_{m i}+M_{t i}\right)}\left(\boldsymbol{v}_{m i 0}-\boldsymbol{v}_{t i 0}\right)^{2} .
$$

According to the above equation, the loss in mechanical energy decreases as the mass of the contact points decreases,

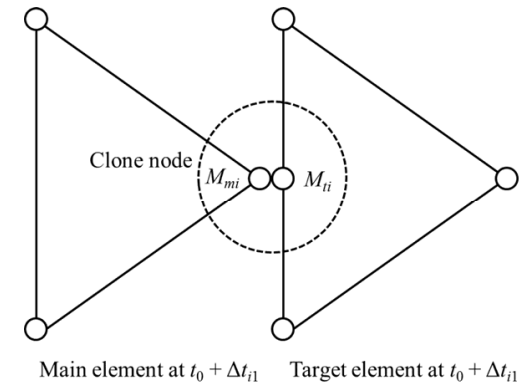

Figure 7 The clone node between two blocks after collision.

and $\Delta E$ is zero if the mass is zero, which generates a completely elastic collision. We can achieve collisions with different energy losses by adjusting the mass of the contact points. As the mass of the contact points is identified, the masses of other nodes may be acquired according to traditional methods.

In fact, the assumption of a completely inelastic collision is reasonable if we consider the colliding body as an assembly consisting of many very thin layers parallel to the contact face during a real collision. The layer is thin and its mass is very small, so even a small force can make it accelerate very quickly. The normal velocity of the surface layers adjacent to the impact point becomes the same as soon as the collision occurs. This process is truly and completely inelastic.

\subsection{Process from $t_{0}+\Delta t$ till the time of separation}

The clone node still exists at $t_{0}+\Delta t$. During the following simulation, it is treated as a continuous node until the normal contact force satisfies

$$
-F_{c}^{n}(\tau+\Delta \tau)>\sigma_{t} A_{c} .
$$

Thus, both the normal and shear forces are set to zero. In the above formula, $\sigma_{t}$ is the contact tensile strength and $A_{c}$ is the contact area. Otherwise, the maximum shear force is calculated as

$$
F_{c \max }^{s}=F_{c}^{n} \tan \varphi+c A_{c},
$$

where $\varphi$ is the friction angle and $c$ is the contact cohesion. If the absolute value of the shear force given by

$$
F_{c}^{s}(\tau+\Delta \tau)=\sqrt{F_{c i}^{s}(\tau+\Delta \tau) F_{c i}^{s}(\tau+\Delta \tau)}
$$

is greater than the maximum shear force, i.e.,

$$
F_{c}^{s}(\tau+\Delta \tau) \geqslant F_{c \max }^{s}
$$

and the shear force is therefore reduced to the limiting value as follows:

$$
F_{c i}^{s}(\tau+\Delta \tau)=F_{c i}^{s}(\tau+\Delta \tau)\left(F_{c \max }^{s} / F_{c}^{s}(\tau+\Delta \tau)\right) .
$$

Since the contact spring has not been introduced, the in- 
teraction forces between contact points instead of spring force are calculated according to eq. (11) (Figure 8).

$$
\left\{\begin{array}{l}
\boldsymbol{F}_{i}=M_{i} \boldsymbol{a}_{i}, \\
\boldsymbol{F}_{m t}+\boldsymbol{F}_{t i}=M_{t i} \boldsymbol{a}_{i},
\end{array}\right.
$$

where $\boldsymbol{a}_{i}$ is the acceleration of the clone node, and $\boldsymbol{F}_{m t}$ is the force between contact point $m$ and contact point $t$. Thus, $\boldsymbol{F}_{m t}$ is decomposed along the normal and the tangents as the normal and shear forces used for the above criteria. If the criterion in either eq. (6) or eq. (9) is satisfied, then the clone node is removed and the subdivided elements will be deleted.

\section{The concept of two space scale elements}

In the unified node model described in the previous section, contact forces are calculated according to the deformation of the main and target elements. When a collision occurs, the elastic wave will propagate from the contact nodes. Let the velocity of elastic wave be denoted as $c$. As mentioned above, during the contact moment in one calculation step, the real contact time is $\Delta t_{i 2}$, which is shorter than $\Delta t$. The region for the propagation of this wave may be denoted as $c \Delta t_{i 2}$, which is the characteristic length of the collision. Therefore, $c \Delta t_{i 2}$ is smaller than $c \Delta t$. Let $\Delta x_{i}$ be the characteristic length of the element. In order to maintain the convergence of an explicit time-history analysis approach, $c \Delta t$ must be smaller than $\Delta x_{i}$. Therefore, $c \Delta t_{i 2}$ is smaller than $\Delta x_{i}$ accordingly, which means that the accurate range affected by the collision is smaller than $c \Delta t$ and also smaller than $\Delta x_{i}$ (Figure 9). However, the region used to calculate contact forces in the current method measures one element length, which is larger than the real zone and leads to a lower stiffness value and a larger mass. Furthermore, this will lead to an inaccurate calculation of contact forces.

In order to precisely describe the process of propagation of elastic waves and obtain more accurate contact forces, an approach of two space scales has been developed. In this new approach, two colliding elements are subdivided after $t_{0}+\Delta t_{i 1}$ based on the collision characteristic length, $c \Delta t_{i 2}$. The subdivided elements are calculated independently, and

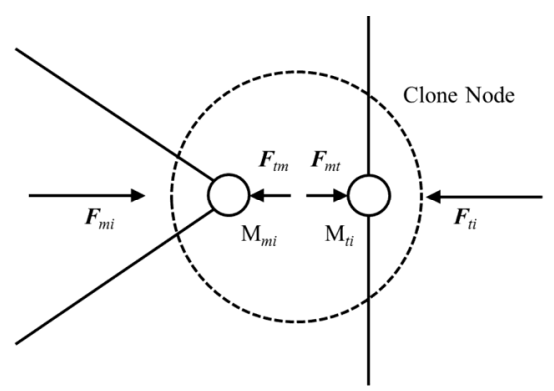

Figure 8 Interaction forces between contact points

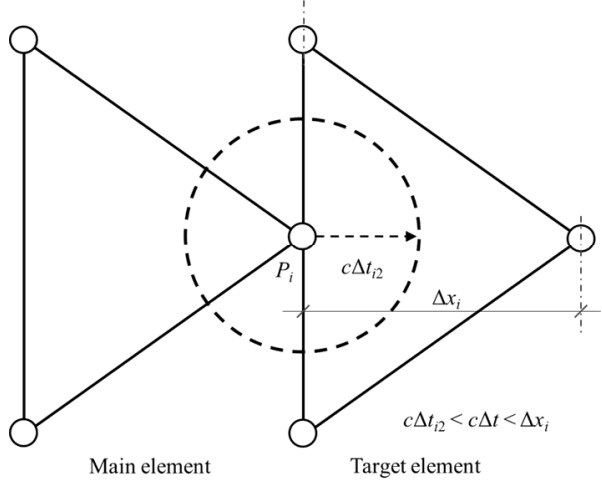

Figure 9 Characteristic length of the collision, denoted as $c \Delta t_{i 2}$.

the interactions with other elements are calculated using the initial nodes of the two elements. In summary, the node velocities of subdivided elements in the present step are obtained from the initial node information of the two elements. In this manner, the simulation of subdivided elements may be treated as an initial value problem of continuous mechanics with velocity boundary conditions. Based on the results of the calculation from subdivided elements, element forces are transferred to the initial nodes of the two elements. In order to satisfy the convergence condition, calculation steps of the subdivided elements may be adapted.

Specifically, subdivisions will only be implemented in the elements where collisions occur during time interval $t_{0}+$ $\Delta t_{i 1}$ to the time of separation of the contact points. In this manner, local subdivisions will be employed so as to not influence the resolution of the entire region. The procedures for two-space scales may be described as follows.

(1) $P_{i}$ represents the center of the circle, and $c_{m i} \Delta t_{i 2}$ and $c_{t i} \Delta t_{i 2}$ represent the radii. Circles $O_{m i}$ and $O_{t i}$ may be obtained. The characteristic velocity, $c_{m i}$ or $c_{t i}$, of elastic waves in the element may be identified as $\sqrt{E / \rho}$ for each element. The region of subdivisions is shown in Figure 10.

(2) For each colliding element, all of the initial nodes are calculated using the time step $\Delta t$, and its velocities may be obtained.

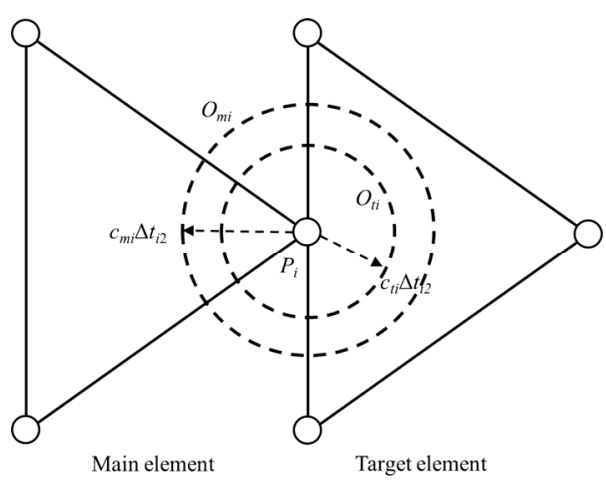

Figure 10 Process of identifying the refined zone. 
(3) Transfer the velocity of the initial nodes to their corresponding inside nodes, and fix the velocity of these nodes. The solution of the subdivided elements may then be regarded as an initial value problem of continuous mechanics with velocity boundary conditions, as shown in Figure 11. The equation for this problem may be described as follows:

$$
m_{j} \frac{\mathrm{d}^{2} \boldsymbol{u}_{j}}{\mathrm{~d} t^{2}}=\sum_{k} K_{k j} \boldsymbol{u}_{k},
$$

where $j$ is a node belonging to the sub-element, $m_{j}$ is the mass of the node, $k$ is the related node, and $\boldsymbol{u}_{i}$ and $\boldsymbol{u}_{j}$ are the displacements of $i$ and $j$, respectively. $K_{i j}$ is the stiffness coefficient matrix for nodes $k$ to $j$, which is an accumulation of the corresponding coefficients of related element stiffness matrices. In general, as the subdivided elements are defined according to $c_{t i} \Delta t_{i 2}$, the coordinates of these elements will include this particular characteristic length. That is to say that $c_{t i} \Delta t_{i 2}$ will appear in $K_{k j}$, and different collisions will present different formats.

(4) Solve eq. (12) with a time step of $\Delta t / n$ and duration of $\Delta t$. Then, the forces of the element on the subdivided elements may be obtained.

(5) Transfer the forces of the elements to the initial nodes, and the force values for the whole element may be acquired.

\section{Verification examples}

The algorithm described in the previous sections was implemented using a $\mathrm{VC}++$ program. An example is shown in the following section.

As shown in Figure 12, this case involves a system of two triangular prism blocks. Both of them are free, and block $\mathbf{i}$ moves towards and impacts block $\mathbf{j}$. The values of Young's modulus, Poisson's ratio, and mass density for each block are $E=40 \mathrm{GPa}, v=0.25$, and $\rho=2700 \mathrm{~kg} / \mathrm{m}^{3}$, respectively. The time step is $5 \times 10^{-4} \mathrm{~s}$. The tolerance of the contact search is $5 \times 10^{-3} \mathrm{~m}$. The initial velocity of block $\mathbf{i}$ is $3.0 \mathrm{~m} / \mathrm{s}$, and that of block $\mathbf{j}$ is $0.0 \mathrm{~m} / \mathrm{s}$ in the $x$-direction. Their initial distance is $1 \mathrm{~m}$ in the $x$-direction.

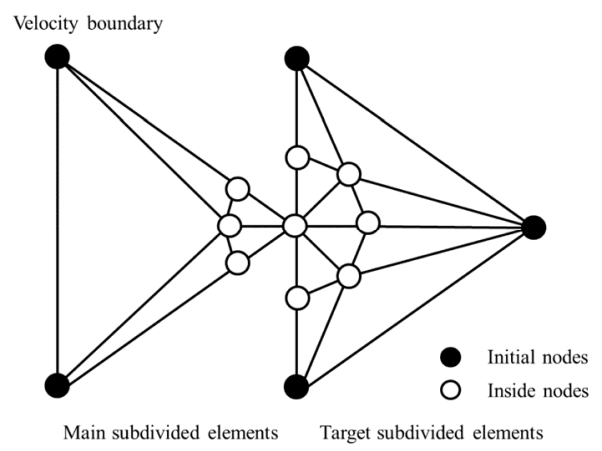

Figure 11 Subdivided elements during the collision.

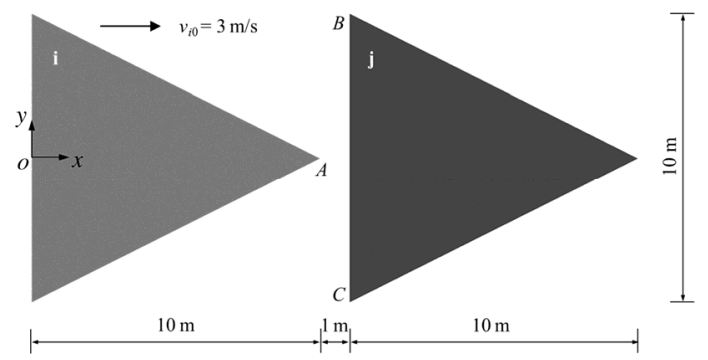

Figure 12 Geometric model of two colliding blocks.

Figure 13 shows the motion process from $0.0 \mathrm{~s}$ to the precise moment when the collision occurs. As shown in the figure, block $\mathbf{i}$ moves towards block $\mathbf{j}$ at a constant velocity. At a time of $0.3333 \mathrm{~s}$, vertex A of block $\mathbf{i}$ touches the edge of block $\mathbf{j}$. Here, the time of $0.3333 \mathrm{~s}$ is in the step from 0.3335 to $0.3340 \mathrm{~s}$, and its value is obtained using the twostage approach mentioned above.

After the precisely touched moment at $0.3333 \mathrm{~s}$, the unified node and subdivided elements are established to calculate the contact forces. As shown in Figure 14, the illustrated collision process is from time 0.3335 to $0.3400 \mathrm{~s}$. The unified node exits during the process, and subdivided elements are used to calculate the interaction between block $\mathbf{i}$ and block $\mathbf{j}$. In addition, the propagation of elastic waves during the process may also be observed from this result. In this stage, contact forces act on block $\mathbf{j}$, which undergoes an acceleration process. The velocity of block $\mathbf{j}$ is increased from 0.0 to $3.205 \mathrm{~m} / \mathrm{s}$ (Figure 14(c)).

As block $\mathbf{j}$ accelerates and block $\mathbf{i}$ decelerates, the criteria established for the unified node will come into effect. If the criteria are satisfied, then the unified node will be separated, which can be seen in Figure 15.

The resulting displacement in the $\mathrm{x}$-direction of the two elements is shown in Figure 16, and may be used to quantitatively analyze the numerical results. From the gradients of these two displacement curves, we may conclude that the velocity of block $\mathbf{j}$ after collision is approximately equal to the initial velocity of block i. A little energy loss occurs
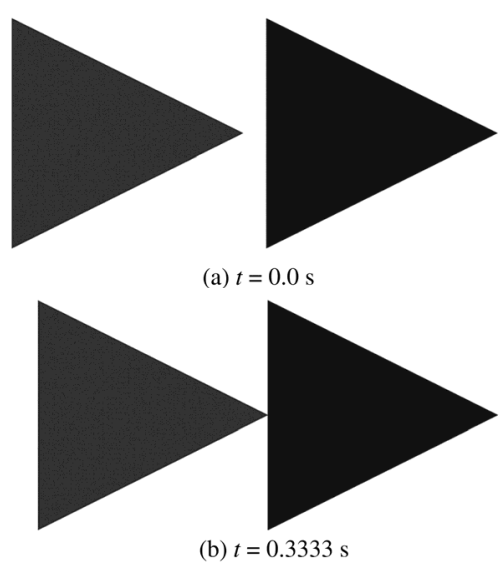

Figure 13 Geometric model of two colliding blocks. 

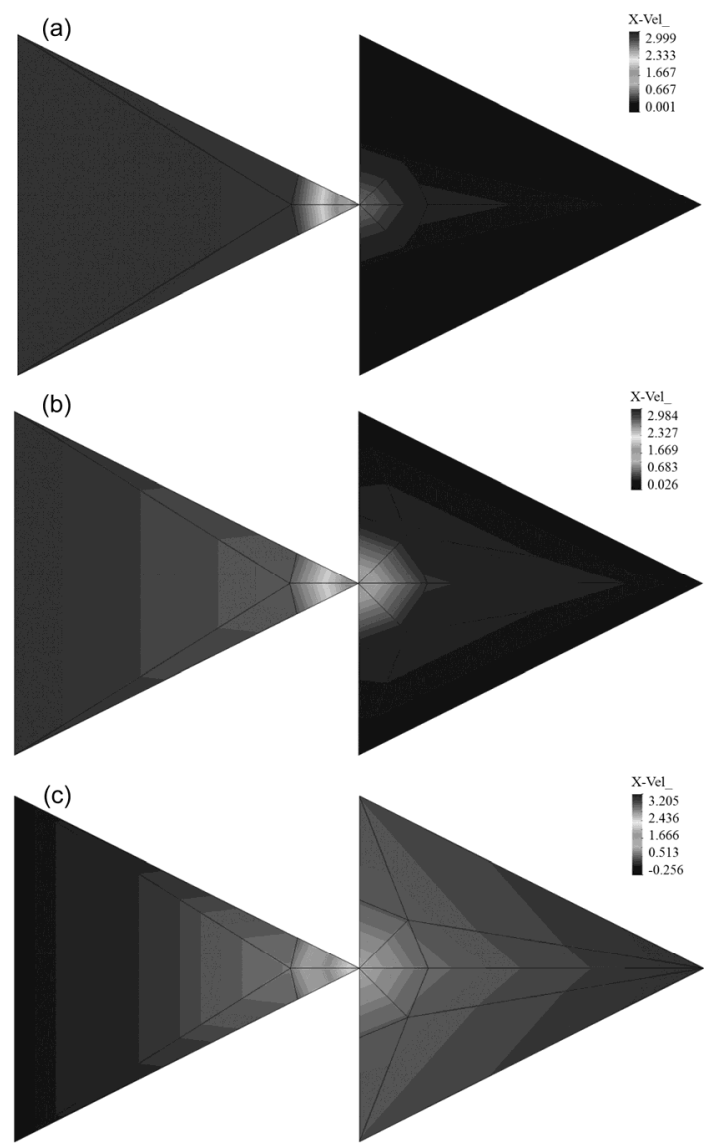

Figure 14 Collision process calculated using the unified node and subdivided elements. (a) $0.3335 \mathrm{~s}$; (b) $0.3340 \mathrm{~s}$; (c) $0.3400 \mathrm{~s}$.
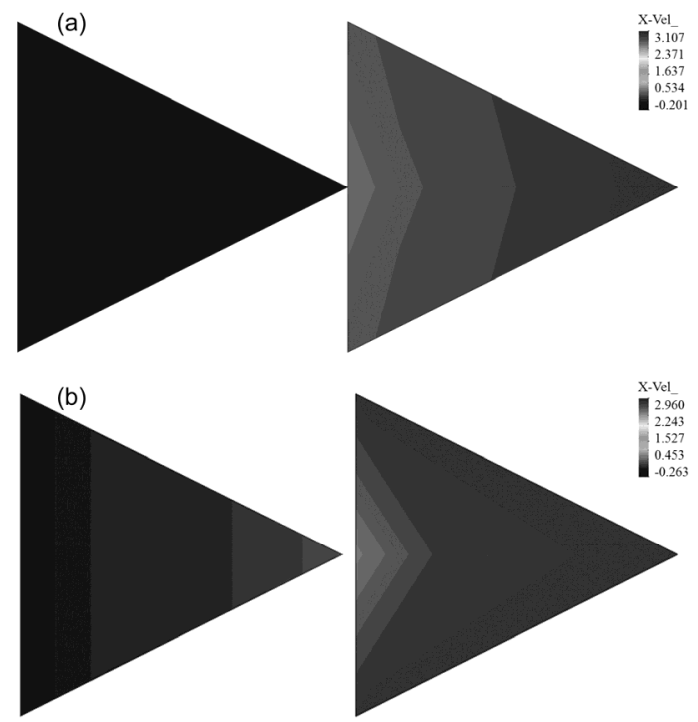

Figure 15 Separation of the unified node, and the two blocks. (a) 0.3405 s; (b) $0.4960 \mathrm{~s}$

during the collision process, as a result of momentum conservation applied. This is reasonable when considering true physical processes.

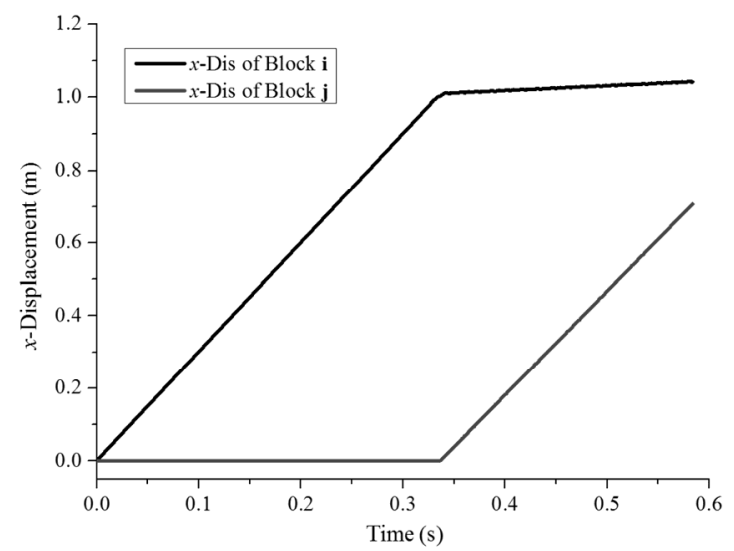

Figure 16 Displacement in the $x$-direction of the two blocks during collision.

\section{Conclusion}

In this study, the precise time of collision was considered to be a characteristic time, and the precise range of the elastic wave caused by collision was considered to be a characteristic length. A new contact model was presented to divide the time step of the contact node and refine the elements adjacent to the contact point according to this characteristic length. Finally, a method for calculating the contact forces without contact springs was introduced to solve the collision problem. A combination of these two methods provided a new way to solve the problem of the scale of the elements involved in a collision and to assess the issues caused by contact springs, which are of great importance.

The idea of a two-space scale contact model with no use of mathematical springs was a new way to simulate contact processes. In this study, emphasis was placed on developing the two-space scale contact model systematically, rather than through accomplishment of all the details. Nevertheless, more work needs to be done to study and further research these details for engineering problem applications.

These works mainly include the control of local mesh equality, the mesh refinement algorithm when there are many contact nodes, tangential sliding contact nodes, and the parallel algorithm for local mesh refinement.

This work was supported by the National Basic Research Program of China ("973" Project) (Grant No. 2015CB250903), the CAS Strategic Priority Research Program (B) (Grant No. XDB10030303), the National Key Technology Research and Development Program of the Ministry of Science and Technology of China (Grant No. 2012BAK10B01), and the Youth Science Fund of the National Natural Science Foundation of China (Grant No. 11302230).

1 Cundall P A. Formulation of a three-dimensional distinct element model-Part I. A scheme to detect and represent contacts in a system composed of many polyhedral blocks. Int J Rock Mech Min, 1988, 25: $107-116$

2 Hart R, Cundall P A, Lemos J. Formulation of a three-dimensional 
distinct element model-Part II. Mechanical calculations for motion and interaction of a system composed of many polyhedral blocks. Int J Rock Mech Min, 1988, 25: 117-125

3 Williams J R, O'Connor R. A linear complexity intersection algorithm for discrete element simulation of arbitrary geometries. Eng Comput, 1995, 12: 185-201

4 Shi G H. Discontinuous deformation analysis-A new numerical model for the statics and dynamics of block systems. Dissertation of Doctor Degree. Dept Civ Eng, UC Berkeley, 1988

5 Wu J H, Ohnishi Y, Shi G H, et al. Theory of three-dimensional discontinuous deformation analysis and its application to a slope toppling at Amatoribashi, Japan. Int J Geomechanics, 2005, 5: 179-195

6 Ning $Y$, Yang J, An X, et al. Modelling rock fracturing and blast-induced rock mass failure via advanced discretisation within the discontinuous deformation analysis framework. Comput Geotech, 2011, 38: 40-49

7 Beyabanaki S A R, Jafari A, Biabanaki S O R, et al. Nodal-based three-dimensional discontinuous deformation analysis (3-D DDA). Comput Geotech, 2009, 36: 359-372

8 Munjiza A, Owen D R J, Bicanic N. A combined finite-discrete element method in transient dynamics of fracturing solids. Eng Computation, 1995, 12: 145-174

9 Munjiza A, Andrews K R F. Penalty function method for combined finite-discrete element systems comprising large number of separate bodies. Int J Numer Meth Eng, 2000, 49: 1377-1396

10 Shi G H. Manifold method of material analysis. In: Proceedings of the Trans Ninth Army Conference on Applied Mathematics and Computing, Minneapolis, 1991. 57-76

11 Wu Z, Wong L N Y. Frictional crack initiation and propagation analysis using the numerical manifold method. Comput Geotech, 2012, 39: $38-53$
12 Lankarani H M, Nikravesh P E. A contact force model with hysteresis damping for impact analysis of multibody systems. J Mech Design, 1990, 112: 369-376

13 Lankarani H M, Nikravesh P E. Continuous contact force models for impact analysis in multibody systems. Nonlinear Dynam, 1994, 5: 193-207

14 He L, An X M, Ma G W, et al. Development of three-dimensional numerical manifold method for jointed rock slope stability analysis. Int J Rock Mech Min, 2013, 64: 22-35

$15 \mathrm{Wu} \mathrm{J} \mathrm{H.} \mathrm{New} \mathrm{edge-to-edge} \mathrm{contact} \mathrm{calculating} \mathrm{algorithm} \mathrm{in} \mathrm{three-}$ dimensional discrete numerical analysis. Adv Eng Softw, 2008, 39: $15-24$

16 Beyabanaki S A R, Mikola R G, Hatami K. Three-dimensional discontinuous deformation analysis (3-D DDA) using a new contact resolution algorithm. Comput Geotech, 2008, 35: 346-356

17 Yeung M R, Jiang Q H, Sun N. A model of edge-to-edge contact for three-dimensional discontinuous deformation analysis. Comput Geotech, 2007, 34: 175-186

18 Jing L. A review of techniques, advances and outstanding issues in numerical modelling for rock mechanics and rock engineering. Int $\mathbf{J}$ Rock Mech Min, 2003, 40: 283-353

19 Goodman R E, Taylor R L, Brekke T. A model for the mechanics of jointed rock. J Soil Mech Foundations Division, ASCE, 1968, 94: 637-659

20 Zhou D, Li S H, Zhang Q B, et al. Structure layer model in continuum-discontinuum element method. In: Proceedings of the 6th International Conference on Discrete Element Methods and Related Techniques, Colorado, 2013. 445-450

21 Wang J, Li S H, Feng C. A shrunken edge algorithm for contact detection between convex polyhedral blocks. Comput Geotech, 2014, 63: $315-330$ 